ఠ

\title{
miR-376a inhibits breast cancer cell progression by targeting neuropilin-I NR
}

This article was published in the following Dove Press journal:

OncoTargets and Therapy

\author{
Lansheng Zhang ${ }^{1,2}$ \\ Yanwei Chen ${ }^{3}$ \\ Hui Wang ${ }^{3}$ \\ Xia Zheng ${ }^{2}$ \\ Caihong $\mathrm{Li}^{2}$ \\ Zhengxiang $\mathrm{Han}^{3}$
}

'Department of Radiation Oncology, Shandong Cancer Hospital Affiliated to Shandong University, Jinan, People's Republic of China; ${ }^{2}$ Department of Radiation Oncology, the Second Affiliated Hospital of Xuzhou Medical University, Xuzhou, People's Republic of China; ${ }^{3}$ Department of Oncology, The Affiliated Hospital of Xuzhou Medical University, Xuzhou, People's Republic of China
Correspondence: Zhengxiang Han Department of Oncology, the Affiliated Hospital of Xuzhou Medical University, No. 99 huaihai west road, Xuzhou 221000, China

Tel +865I6858020II

Email zhxh_xmu@I63.com
Background: The roles and related mechanism of miR-376a in breast cancer cell progression are unclear.

Methods: Kaplan-Meier plotter analysis was used to analyze the correlation between miR-376a and the overall survival (OS) of breast cancer patients. Quantitative reverse transcription polymerase chain reaction (qRT-PCR) was performed to detect miR-376a level in breast cancer cells. Cell viability, transwell migration and invasion, and cell apoptosis were constructed to investigate the effects of miR-376a on breast cancer cells. Luciferase reporter and RNA immunoprecipitation (RIP) were used to explore the targeting of miR-376a on NRP-1.

Results: miR-376a expression was positively correlated with the overall survival of breast cancer patients, and significantly decreased in breast cancer cells. Functionally, miR-376a overexpression suppressed cell proliferation, migration and invasion, and promoted cells apoptosis. Additionally, miR-376a could directly target NRP-1 and exerted its effect through NRP-1. Conclusion: miR-376a could suppress breast cancer cell progression via directly targeting NRP-1. Keywords: miR-376a, NRP-1, breast cancer, Wnt/ $\beta$-catenin migration

\section{Introduction}

Breast cancer is the most common cancer in women worldwide. ${ }^{1}$ Based on the expression profiles, breast cancer is divided into Luminal A, Luminal B, Her2 high expression and Basal-like subtypes, all of which have their specific tumor biological characteristics. $^{2}$ Although endocrine therapy, Her2-targeted, CDK4/6 inhibitor and chemotherapy have greatly improved the survival of breast cancer patients, the drug resistance and recurrence are still the main obstacles in clinical treatment. ${ }^{3}$ Therefore, it is necessary to find novel targets, markers or methods for breast cancer treatment.

Neuropilin 1 (NRP-1) has been proven to be highly expressed in various tumors, such as lung, gastric and breast cancers. ${ }^{4-6}$ A previous study has indicated that NRP-1 is an associated molecule in the blood, which distinguishes poor prognosis of breast cancer. ${ }^{7}$ NRP-1 is an angiogenic co-receptor of VEGF-A, and VEGF-A/NRP-1 axis could promote breast cancer progression via enhancement of epithelial-mesenchymal transition (EMT) and activation of NF- $\kappa \mathrm{B}$ and $\beta$-catenin signaling. ${ }^{8}$ Notably, a previous research has reported that peptides inhibiting the binding of VEGF-A/NRP-1 could inhibit breast cancer progression, ${ }^{9}$ and two groups have demonstrated that a monoclonal antibody targeting neuropilin-1 or a neuropilin-1 antagonist could exert anticancer effects in breast cancer via in vitro and in vivo experiments. ${ }^{10,11}$ Our studies have previously shown that RNA interference-mediated NRP-1 silencing could inhibit breast cancer cell proliferation and promote cell apoptosis, and VEGF-A/NRP-1 pathway could confer cancer stemness via activating $\mathrm{Wnt} / \beta$-Catenin axis in breast 
cancer cells. ${ }^{12,13}$ However, the mechanisms by which NRP-1 is regulated in breast cancer cells are still not clear.

miRNAs are a kind of noncoding RNA, which could inhibit the expression of transcripts by directly binding to transcripts and thus promoting their degradation or inhibiting their translation. ${ }^{14}$ Various studies have indicated that miRNAs hold critical roles in tumor progression, for example, Muhammad et al ${ }^{15}$ demonstrated that anti-miR-203 suppresses breast cancer growth and stemness by targeting SOCS3 and $\mathrm{Hu}$ et $\mathrm{al}^{16}$ showed that miR-125b inhibits acute myeloid leukemia cell differentiation by directly targeting Fes. A previous study has indicated that miR-376a could regulate proliferation, apoptosis, migration and invasion in metastatic prostate cancer cells. ${ }^{17}$ miR-376a serves as a potential diagnostic and prognostic marker in ovarian cancer patients ${ }^{18}$ and human gliomas. ${ }^{19}$ It also acts as a tumor suppressor in non-small-cell lung cancer ${ }^{20}$ and giant cell tumor of bone. ${ }^{21}$ However, there is no evidence reporting the roles and related mechanisms in breast cancer progression.

In this study, we first explored the correlation between miR-376a expression and the overall survival (OS) of breast cancer patients, and we found that miR-376a expression was positively correlated with the OS of breast cancer patients, which indicates the inhibitory role of miR-376a in breast cancer. Furthermore, cell viability, cell apoptosis, migration and invasion assays were performed to prove our speculation. Finally, our results demonstrate that miR-376a could directly target to NRP-1 and inactivate the downstream axis Wnt/ $\beta$-catenin. Thus, we concluded that miR-376a acts as a tumor suppressor via targeting NRP-1 in breast cancer.

\section{Materials and methods Kaplan-Meier (KM) plotter analysis}

KM plotter analysis was done to evaluate the correlation between miR-376a expression and the OS of breast cancer patients following the previous study. ${ }^{22}$ Breast cancer METABRIC and GSE19783 datasets were used for this analysis, which include 1,262 and 101 breast cancer patients, respectively, and the cutoff was autoselected.

\section{Cell cultures and reagents}

Breast cancer cell lines MCF-7, MDA-MB-453 and MDAMB-231 and breast epithelial cells MCF-10A were purchased from the China Academia Sinica Cell Repository (Shanghai, China). MCF-10A, MCF-7 and MDA-MB-453 cells were cultured in DMEM (Thermo Fisher Scientific, Waltham, MA, USA), and MDA-MB-231 cells were maintained in L-15 medium (Thermo Fisher Scientific) supplemented with $10 \%$ fetal bovine serum (FBS; Thermo Fisher Scientific),
$80 \mathrm{U} / \mathrm{mL}$ penicillin and $0.08 \mathrm{mg} / \mathrm{mL}$ streptomycin at $37^{\circ} \mathrm{C}$ under humidified atmosphere with $5 \% \mathrm{CO}_{2}$. Wnt $/ \beta$-catenin agonist, SKL2001 (S8320), was purchased from Selleck.cn (Houston, TX, USA).

\section{Lentivirus package and stable infection cell line construction}

The NRP-1 overexpression lentivirus vector was constructed in our previous study and referred to as LV-NRP-1. ${ }^{12} \mathrm{We}$ thank RiboBio Inc. (Guangzhou, China) for constructing miR-376a overexpression lentivirus vector, LV-miR-376a and miR-control lentivirus (control). For constructing miR-376a stable overexpression cells, MDA-MB-453 and MDA-MB-231 cells were infected with LV-miR-376a virus or control virus in the presence of $2 \mu \mathrm{g} / \mathrm{mL}$ of polybrene. After 48 hours, the cells were selected with $1.5 \mu \mathrm{g} / \mathrm{mL}$ of puromycin (Sigma-Aldrich Co., St Louis, MO, USA) for 3 weeks. Furthermore, quantitative real-time PCR (qRTPCR) was used to examine the infection efficiency of miR$376 \mathrm{a}$ in cells.

\section{Cell viability assay}

MDA-MB-453 and MDA-MB-231 cells were seeded in 96-well plates at $4.0 \times 10^{3}$ cells/well with LV-NRP-1 infection or not. After 24 hours, 48 hours and 72 hours, cell viability was detected using CCK8 assay (MedChemExpress, Cat No: HY-K0301) according to the manufacturer's protocols.

\section{Cell apoptosis assay}

MDA-MB-453 and MDA-MB-231 cells with miR-376a stable overexpression were infected with LV-NRP-1 and washed with cold PBS, and then resuspended in the binding buffer containing Annexin V-fluorescein isothiocyanate (FITC) and propidium iodide (PI; Beyotime, Beijing, China) for 15 minutes in the dark at room temperature. Cell apoptosis was then detected by the FACS flow cytometry (BD Biosciences, San Jose, CA, USA).

\section{Cell migration and invasion assay}

The detailed procedure is mentioned in the previous study. ${ }^{23}$ In this study, the measure time of migration and invasion was 24 and 48 hours, respectively.

\section{qRT-PCR assay}

Total RNA was extracted using TransZol Up (TransGen Biotech, Beijing, China) following the manufacturer's protocols. cDNA synthesis and qRT-PCR assay for miR-376a were performed using Hairpin-itTM Real-Time PCR miRNAs kit (GenePharma, Shanghai, China). cDNA synthesis for 
mRNA was performed using EasyScript First-Strand cDNA Synthesis SuperMix (TransGen Biotech), and Custom RealTime PCR Gene Detection Assay (GenePharma) was used to analyze mRNA expression. All qRT-PCR reactions were performed on an ABI Prism 7500 Detection System (Thermo Fisher Scientific). GAPDH was served as an internal control. The relative gene expression was analyzed using the $2^{-\Delta \Delta \mathrm{ct}}$ method.

\section{Western blot}

The detailed procedure is mentioned in our previous study. ${ }^{12}$ The primary antibodies against NRP-1 (ab81321), E-cadherin (ab1416), Vimentin (ab8978), Wnt3a (ab28472), $\beta$-catenin (ab32572), anti-cleaved caspase 3 (ab32042), anti-cleaved PARP (ab32064), Caspase 3 (ab13847), PARP (ab74290) and $\beta$-actin (ab8226) were purchased from Abcam (Cambridge, UK). The secondary antibodies $\operatorname{IgG}(\mathrm{H}+\mathrm{L})$ (HRP-labeled Goat Anti-Mouse $\operatorname{IgG}(\mathrm{H}+\mathrm{L})$ ) (A0216) and $\operatorname{IgG}(\mathrm{H}+\mathrm{L})$ (HRP-labeled Goat Anti-Rabbit IgG(H+L)) (A0208) were purchased from Beyotime. Specific signals were detected using ECL chemiluminescence detection kit (Thermo Fisher Scientific) on Tanon 6200 (Tanon, Shanghai, China). $\beta$-Actin was used as an internal control.

Luciferase reporter assay pMIR-Reporter (Promega Corporation, Fitchburg, WI, USA) was used to introduce the fragments of NRP-1-3'-UTR containing the wild-type (wt) and mutant binding sites (mut) for miR-376a, named as Luc-NRP-1-wt and Luc-NRP-1-mut, respectively. Luc-NRP-1-wt or Luc-NRP-1-mut with $\beta$-gal plasmid was transfected into breast cancer cells with miR-376a overexpression using Lip2000 (Thermo Fisher Scientific) according to the standard procedure. After 72 hours, cells were collected and used for luciferase activity analysis using $\mathrm{ONE}_{-G l{ }^{\mathrm{TM}}+\mathrm{Tox}}$ Luciferase Reporter and Cell Viability Assay kit (E7110) and VivoGlo Luciferin- $\beta$-gal Substrate kit (P1061). The luciferase activity was normalized with the $\beta$-gal activity.

\section{RNA immunoprecipitation (RIP) of Ago2}

The detailed procedure is mentioned in the previous study. ${ }^{24}$ Briefly, MCF-7 and MDA-MB-231 cells transfected with Luc-NRP-1-wt or Luc-NRP-1-mut were lysed with $25 \mathrm{mM}$ Tris-HCl buffer ( $\mathrm{pH} 7.5)$ and $100 \mathrm{U} / \mathrm{mL}$ RNase inhibitor (Sigma-Aldrich Co.), and then incubated with protein-A sepharose beads pre-coated with $3 \mu \mathrm{g}$ anti-Ago 2 antibody or control rabbit IgG for 1.5 hours at $4^{\circ} \mathrm{C}$. The RNA-protein complexes were pulled down by protein $\mathrm{A} / \mathrm{G}$ agarose beads, and RNA was extracted with TransZol Up, and miR-376a level was measured by qRT-PCR.

\section{Statistical analysis}

All data were presented as mean \pm SD. Datasets with only two groups were analyzed using a Student's $t$-test. The differences between the groups were analyzed using one-way ANOVA with the Tukey-Kramer post-test, and $P<0.05$ was considered significant.

\section{Results}

miR-376a is downregulated and predicted to target NRP-I in breast cancer cells

Since other and our groups had previously reported the promotive effects of NRP-1 on breast cancer progression, ${ }^{7,8,12,13}$ here we sought to reveal the mechanisms by which NRP-1 was regulated in breast cancer cells. miRNA.org (http://34.236.212.39/microrna/getMrna. do? gene $=8829 \&$ utr $=20945 \&$ organism $=9606$ ) was used to predict the potential miRNAs that could target NRP-1. miR-376a attracted our attention based on the fact that miR376a held two potential binding sites on NRP-1 3'-UTR (Figure 1A). KM plotter analysis based on METABRIC and GSE19783 datasets showed that miR-376a expression was positively correlated with the OS of breast cancer patients (Figure 1B and C). qRT-PCR was further used to detect the miR-376a level in breast cancer and breast epithelial cells, and results indicated that the miR-376a level was significantly downregulated in breast cancer cells (Figure 1D), which is opposite to the expression pattern of NRP-1 in breast cancer. Notably, miR-376a expression was negatively correlated with the metastatic ability of breast cancer cells (MCF-7, low metastatic ability; MDA-MB-453, median metastatic ability; and MDA-MB-231, high metastatic ability). Here, MDA-MB-453 and MDA-MB-231 cells were chosen for the following study.

\section{miR-376a could directly bind to NRP-I and inactivate the downstream $\mathrm{Wnt} / \beta$ - catenin axis in breast cancer cells}

Then, we continue investigating whether miR-376a could indeed target NRP-1 in breast cancer cells. qRT-PCR and Western blot analyses showed that breast cancer cells with miR-376a stable overexpression exhibited relative lower expression level of NRP-1 than that in control cells without miR-376a overexpression (Figure 2A and B). The infection efficiency of LV-miR-376a was confirmed by qRT-PCR assay (Figure 2C). Further luciferase reporter assay indicated that miR-376a could decrease the activity of LucNRP-1-wt but had no effect on the Luc-NRP-1-mut activity (Figure 2D and E). To confirm the direct interaction between miR-376a and NRP-1 at endogenous levels, we performed 
A

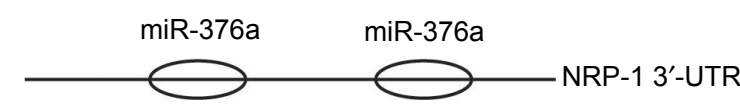

C

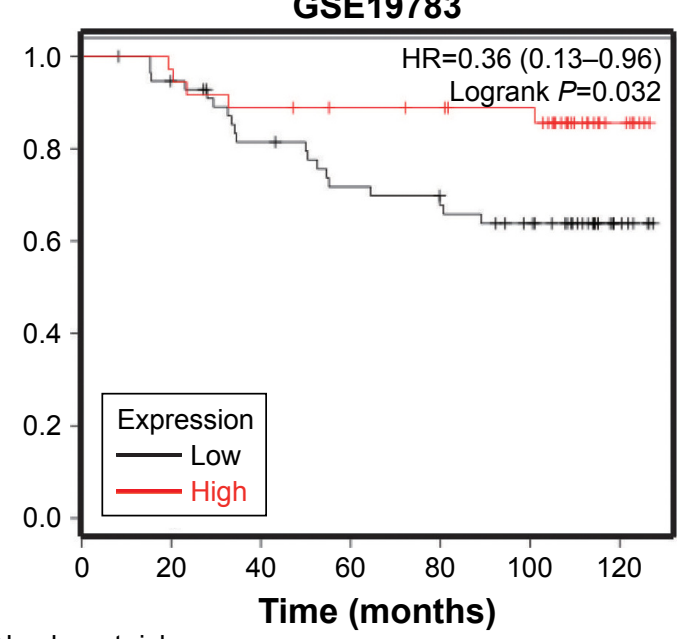

B

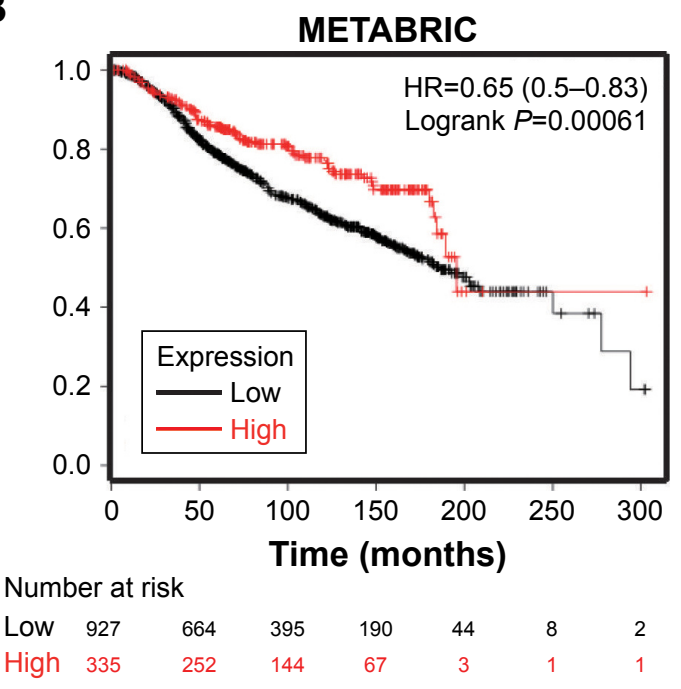

D

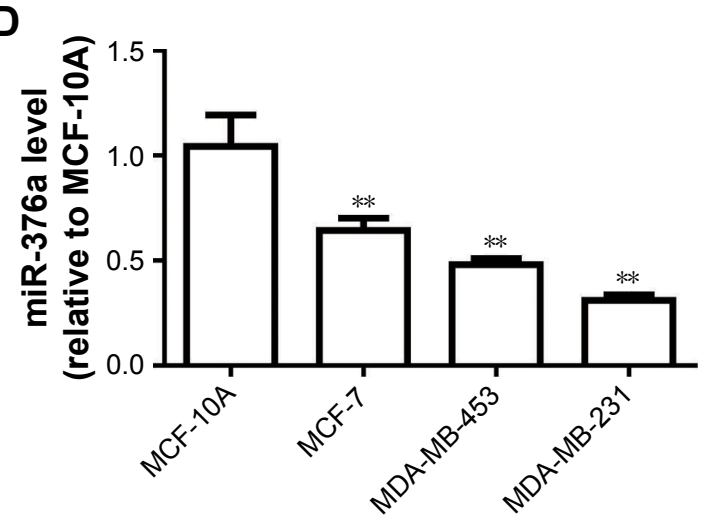

Figure I miR-376a is downregulated and predicted to target NRP-I in breast cancer cells.

Notes: (A) miRNA.org software predicted that miR-376a held two potential binding sites on NRP-I 3'-UTR. (B and C) KM plotter analysis indicated that miR-376a expression was positively correlated with the OS of breast cancer patients based on METABRIC and GSEI9783 datasets. (D) miR-376a level was examined in breast cancer (MCF-7, MDA-MB-453 and MDA-MB-23I) and breast epithelial (MCF-IOA) cells. Data were presented as mean \pm SD; $* * P<0.0$ I vs NC or control.

Abbreviations: KM, Kaplan-Meier; NRP-I, neuropilin-I; OS, overall survival; NC, negative control.

RIP analysis to pull down endogenous miRNAs associated with Ago2 in Luc-NRP-1-wt-overexpressed breast cancer cells. The precipitated miRNAs were subjected to qRT-PCR analysis, and results showed that miR-376a was enriched in RNAs retrieved from Luc-NRP-1-wt-overexpressed cells compared with Luc-NRP-1-mut-overexpressed or control cells (Figure 2F); these results supported that NRP-1 was the bona fide target of miR-376a in breast cancer cells. As we had demonstrated that overexpression of NRP-1 could activate the downstream $\mathrm{Wnt} / \beta$-catenin signaling, we speculated that miR-376a could inactivate this signaling. As expected, miR-376a overexpression significantly decreased Wnt3a and $\beta$-catenin expression in breast cancer cells (Figure 2G). Our results suggested that miR-376a could target NRP-1 and inactivate the downstream signaling in breast cancer cells.

\section{miR-376a overexpression inhibits breast cancer cell proliferation and promotes cell apoptosis}

Based on the correlation between miR-376a and the OS of breast cancer patients, we assumed that miR-376a held inhibitory effects on breast cancer progression. Cell viability assay indicated that miR-376a overexpression inhibited breast cancer cell proliferation (Figure $3 \mathrm{~A}$ and $\mathrm{B}$ ). Further, cell apoptosis analysis showed that overexpression of miR-376a significantly potentiated cell apoptotic rate in breast cancer cells (Figure 3C and D). Additionally, the expression of apoptotic executors (cleaved caspase 3 and cleaved PARP) was examined by Western blot, and consistent results were obtained with an increase in cleaved caspase 3 and cleaved PARP expression (Figure 3E). 

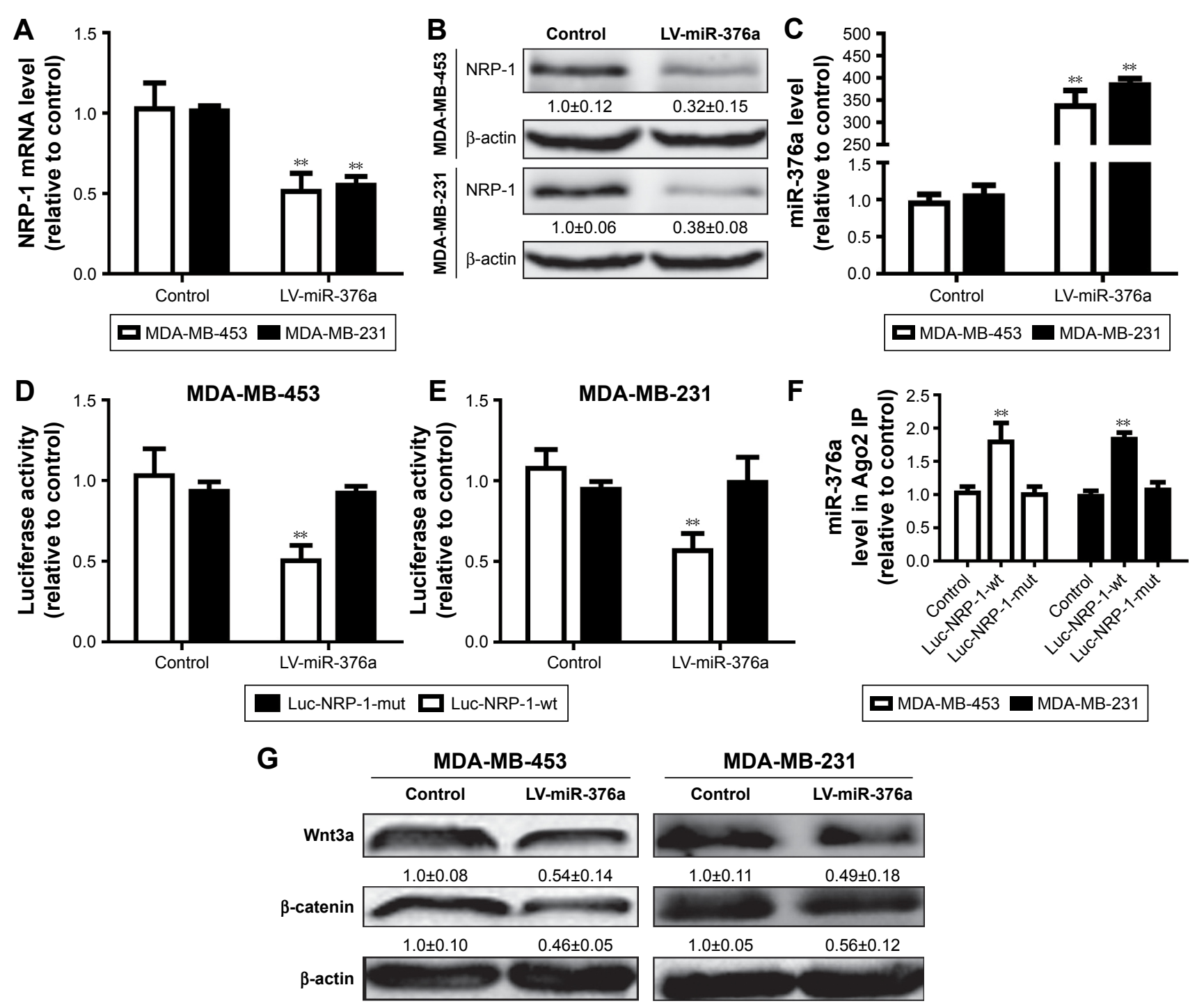

Figure 2 miR-376a could directly bind to NRP-I and inactivate the downstream Wnt/ $\beta$-catenin axis in breast cancer cells.

Notes: (A and B) NRP-I mRNA and protein expression was decreased in MDA-MB-453 and MDA-MB-23I cells with LV-miR-376a infection. (C) The infection efficiency of LV-miR-376a was confirmed by qRT-PCR analysis. (D and E) The luciferase activity of Luc-NRP-mut and Luc-NRP-I-wt was detected in cells depicted in (A). (F) miR-376a level was determined in RNA after being pulled down with anti-Ago2 from breast cancer cells with Luc-NRP-mut or Luc-NRP-I-wt transfection or not. (G) The expression of $W n t 3 a$ and $\beta$-catenin was examined in cells depicted in (A). Data are presented as mean $\pm \mathrm{SD} ; * * P<0.01$ vs $\mathrm{NC}$ or control.

Abbreviations: qRT-PCR, quantitative real-time PCR; mut, mutant; NC, negative control; NRP-I, neuropilin-I; wt, wild-type.

\section{miR-376a overexpression suppresses breast cancer cell migration, invasion and EMT process}

Since miR-376a expression was negatively correlated with the metastatic ability of breast cancer cells, we further explored whether miR-376a could suppress breast cancer migration and invasion. As shown in Figure 4A, MDA-MB-453 and MDA-MB-231 cells with miR-376a stable overexpression exhibited relative lower migration ability compared with control. Consistent results were acquired in the examination of invasion ability (Figure 4B). As EMT process plays a critical role in tumor metastasis, the effects of miR-376a on breast cancer EMT process were detected. We found that miR-376a overexpression decreased the mesenchymal marker (Vimentin) expression, while it increased the epithelial marker (E-cadherin) expression (Figure 4C). Thus, our results demonstrate that miR376a holds inhibitory effects on breast cancer cell progression.

\section{Overexpression of NRP-I or inhibition of wnt/ $\beta$-catenin signaling rescues the inhibitory effects of miR-376a on breast cancer cell progression}

Finally, we further investigated whether the inhibitory effects of miR-376a were dependent on NRP-1 expression and 

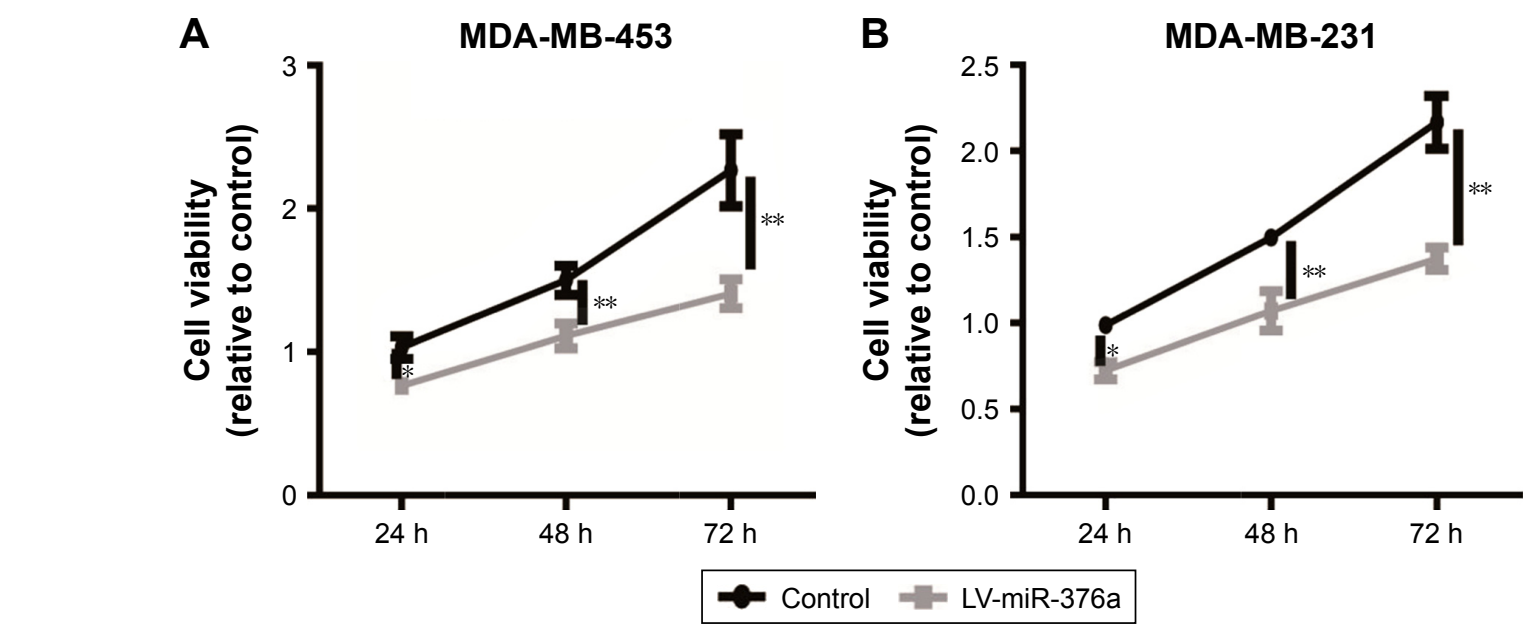

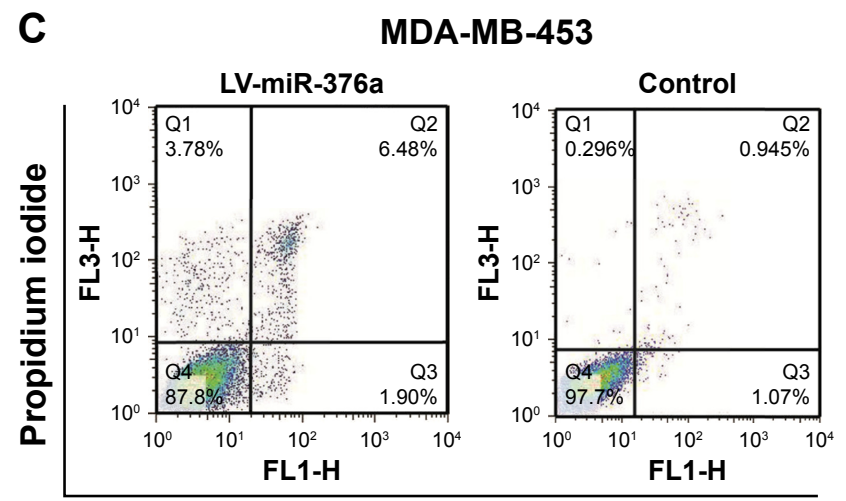

Annexin V-FITC
D

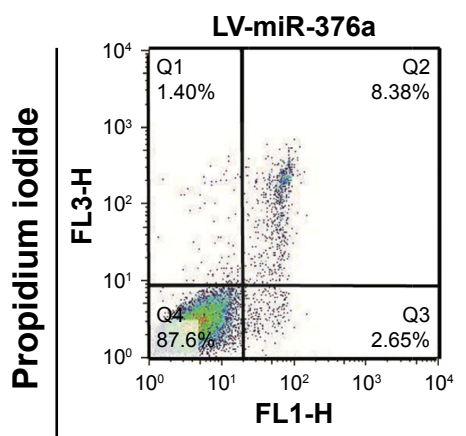

MDA-MB-231

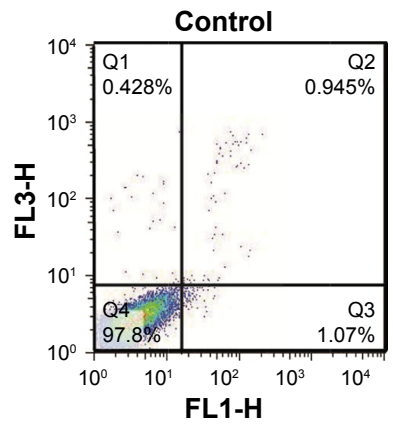

Annexin V-FITC

E

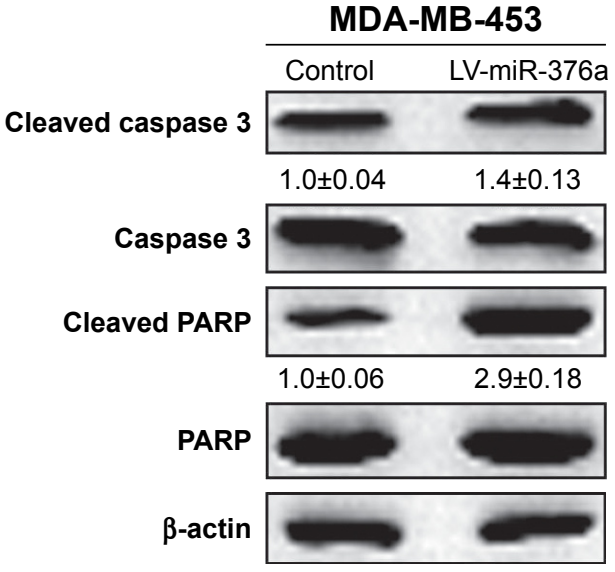

MDA-MB-231

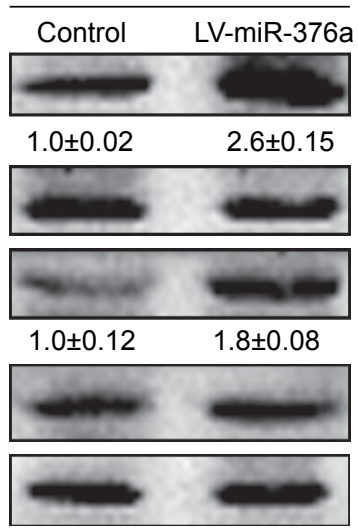

Figure 3 miR-376a overexpression inhibits breast cancer cell proliferation and promoted cell apoptosis.

Notes: (A and B) MDA-MB-453 and MDA-MB-23I cells with miR-376a overexpression or not were subjected to analyze the cell viability by CCK8 assay. (C and D) Cell apoptosis was detected in cells depicted in (A). (E) The expression of apoptosis executors (cleaved caspase 3 and cleaved PARP) was examined in cells depicted in (A). Data are presented as mean $\pm \mathrm{SD} ; * \mathrm{P}<0.05, * * P<0.01$ vs $\mathrm{NC}$ or control.

Abbreviations: FITC, fluorescein isothiocyanate; h, hours; NC, negative control.

downstream Wnt/ $\beta$-catenin signaling. First, cell viability analysis showed that infection with LV-NRP-1 or treatment with Wnt/B-catenin agonist, SKL2001, in miR-376a overexpression cells attenuated the inhibition of miR-376a on cell viability (Figure 5A and B). Furthermore, cell migration assay indicated that overexpression of NRP-1 or SKL2001 treatment rescued miR-376a overexpression-mediated inhibition on breast cancer cell migration and EMT process (Figure 5C and D). Consistent results were obtained in cell apoptosis assay (Figure 5E and F). Therefore, these results 


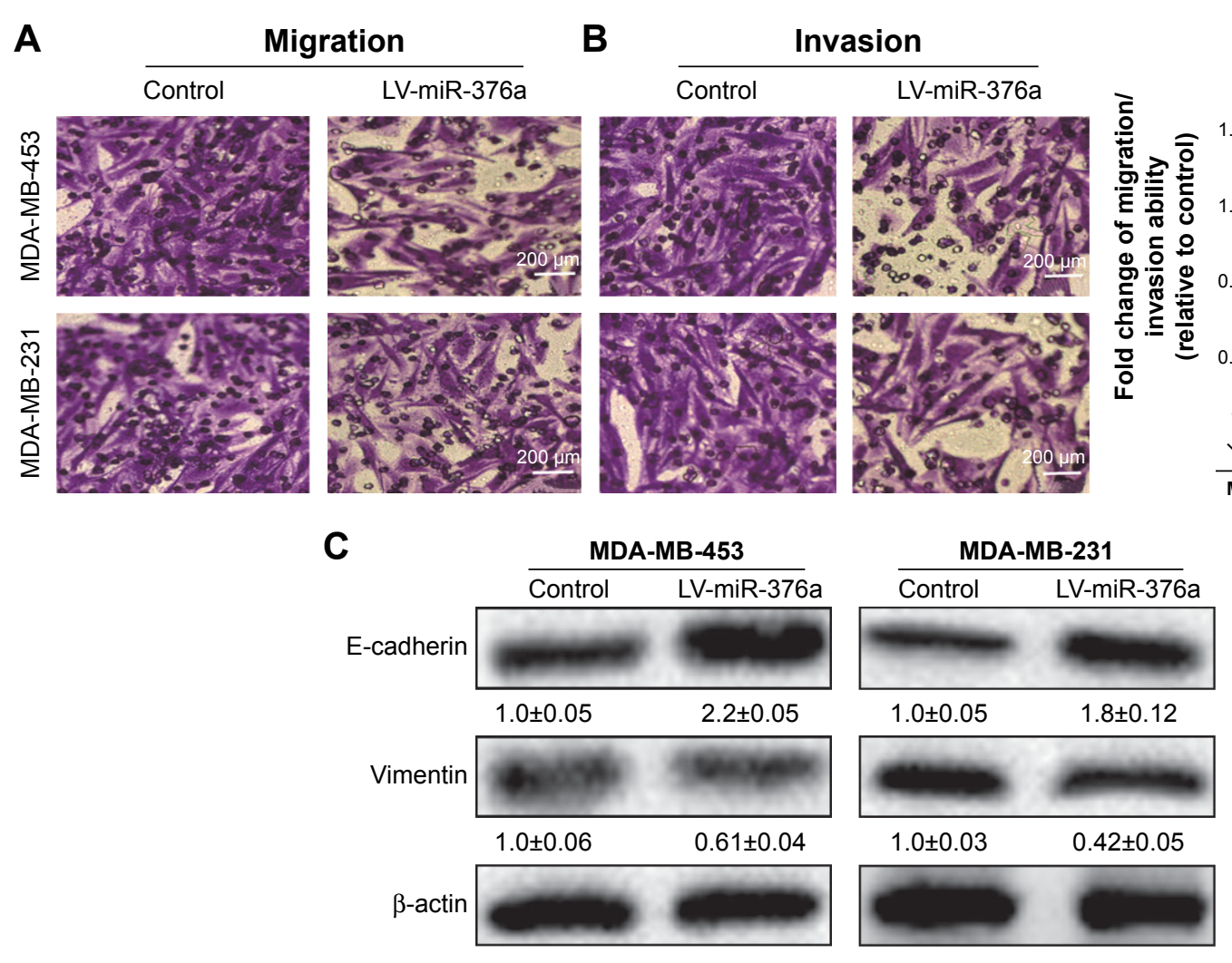

Figure 4 miR-376a overexpression suppresses breast cancer cells migration, invasion and EMT process.

Notes: (A) The migration ability was measured in MDA-MB-453 and MDA-MB-23I cells with miR-376a overexpression or not. (B) The invasion ability was determined in cells depicted in (A). (C) The EMT process was evaluated in cells depicted in (A) via detecting the epithelial marker (E-cadherin) and mesenchymal marker (Vimentin) expression by Western blot assay. Data are presented as mean $\pm \mathrm{SD}$; **P $<0.0$ I vs $\mathrm{NC}$ or control.

Abbreviation: EMT, epithelial-mesenchymal transition; NC, negative control.

suggest that miR-376a overexpression-mediated inhibition on breast cancer cell progression is partly through NRP-1/ $\mathrm{Wnt} / \beta$-catenin axis.

\section{Discussion}

Here, miR-376a inhibitory roles and related mechanisms were first revealed in breast cancer cell progression, which was clarified by the following experiments. First, miR-376a expression was positively correlated with the OS of breast cancer patients, and overexpression of miR-376a inhibited breast cancer cell proliferation, migration, invasion and promoted cell apoptosis. Second, we confirmed that miR-376a could directly bind to NRP-1 and inactivate the downstream Wnt/ $\beta$-catenin signaling. Finally, we indicated that NRP-1 overexpression or treatment with $\mathrm{Wnt} / \beta$-catenin agonist, SKL2001, could rescue the inhibitory effects of miR-376a overexpression on breast cancer cell progression. To the best of our knowledge, this is the first study revealing the roles and related mechanisms of miR-376a in breast cancer.

Other previous studies and this study had indicated the promotive roles of NRP-1 in breast cancer, ${ }^{7,8,12,13}$ however, the mechanisms by which NRP-1 was regulated were unclear. Here, miR-376a was chosen for exploration because it had two potential binding sites on NRP-1 3'-UTR. Notably, another member of miR-376 cluster, miR-376b, was also predicted to hold two potential binding sites on NRP-1 3'-UTR, and miR$376 \mathrm{~b}$ expression was also positively correlated with the OS of breast cancer patients (data not shown). We are not sure whether miR-376b holds similar effects to miR-376a, and whether miR-376a and miR-376b could exert additive effects on breast cancer progression, which should be evaluated in the future works. Based on the critical roles of NRP-1 in breast cancer progression, potential methods targeting NRP-1 were elucidated in several groups; ${ }^{10,11}$ however, the clinical application is still not shown, which might remind us that the mechanisms by which NRP-1 is regulated or NRP-1 functioned still need much more elucidations. In this work, we showed that miR-376a could regulate NRP-1 expression at the transcriptional level, and other miRNAs or other regulatory mechanisms by which NRP-1 was regulated at the translational level should be identified.

Importantly, we must admit that the phenomena and mechanisms identified by us were not confirmed by in vivo 
experiments. Since NRP-1 overexpression or Wnt/ $\beta$-catenin agonist, SKL2001, just could rescue but not reverse the inhibition of miR-376a overexpression on breast cancer cell progression, and one miRNA could target several transcripts, while one transcript could be targeted by several miRNAs. ${ }^{14}$ For example, miR-376a could suppress the proliferation and invasion of non-small-cell lung cancer by targeting c-Myc20 and act as a tumor suppressor by targeting COA1 and PDIA6 in the giant cell tumor of bone. ${ }^{25}$ miRNA-124-3 p/NRP-1 axis plays an important role in mediating glioblastoma growth and angiogenesis. ${ }^{26}$ Therefore, other transcripts, such as c-Myc, COA1 and PDIA6, might involve in miR-376a-mediated inhibition on breast cancer cell progression, and NRP-1 could be regulated by other miRNAs, such as miR-124-3 p, in breast cancer.

Altogether, based on the in vitro experiments in this study, our work reveals an unpredicted layer of NRP-1 regulation and puts the activation of miR-376a as a potential novel therapeutic strategy to specifically inhibit breast cancer progression (Figure 6).
A

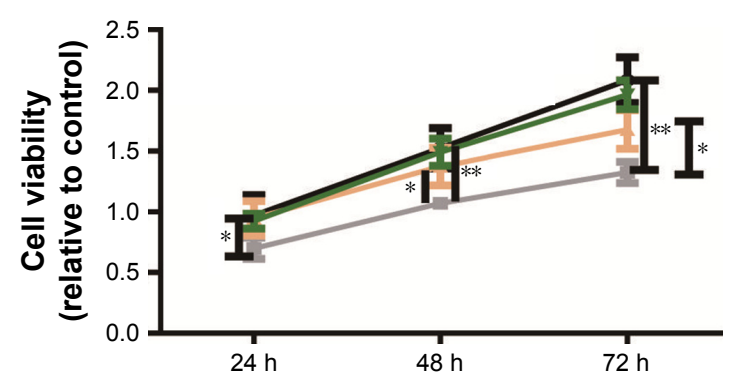

B

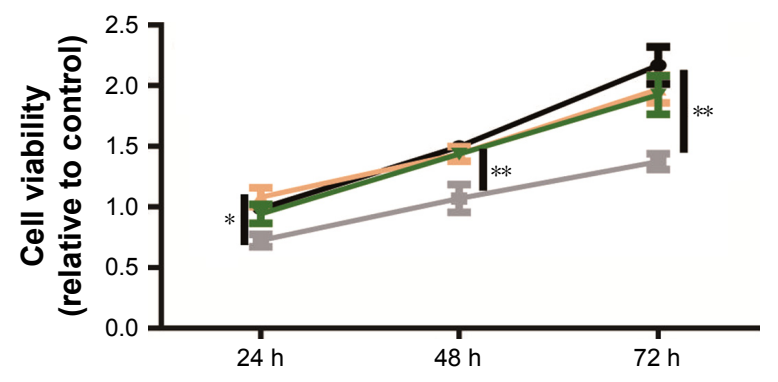

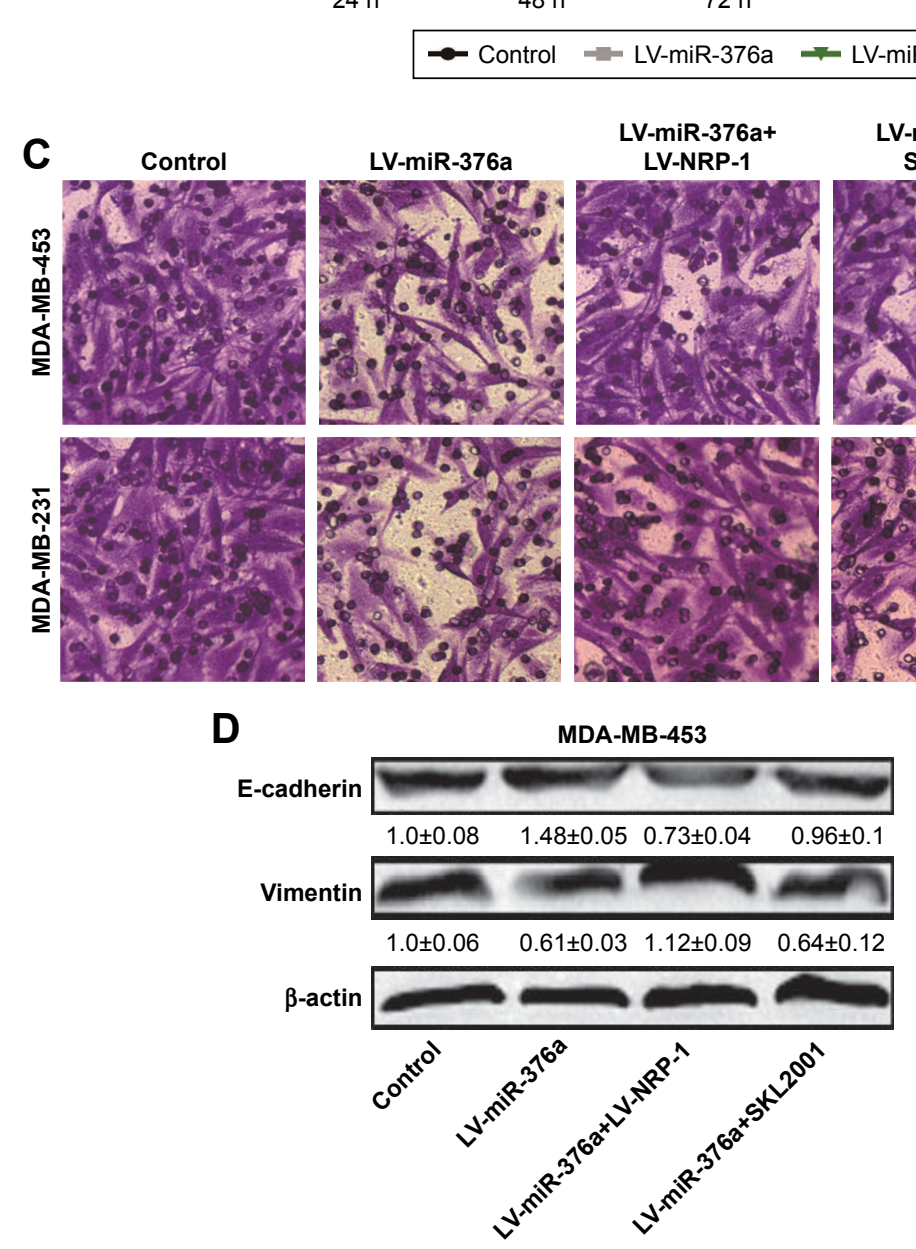

LV-miR-376a+
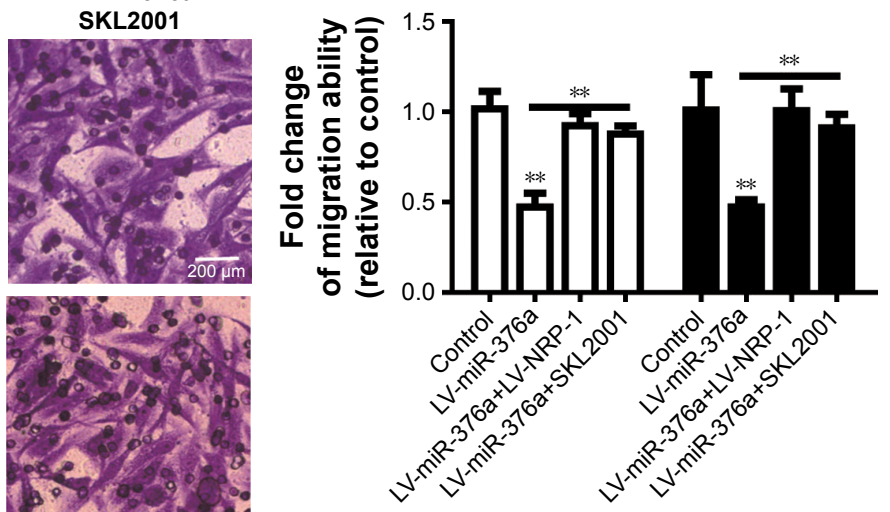

D

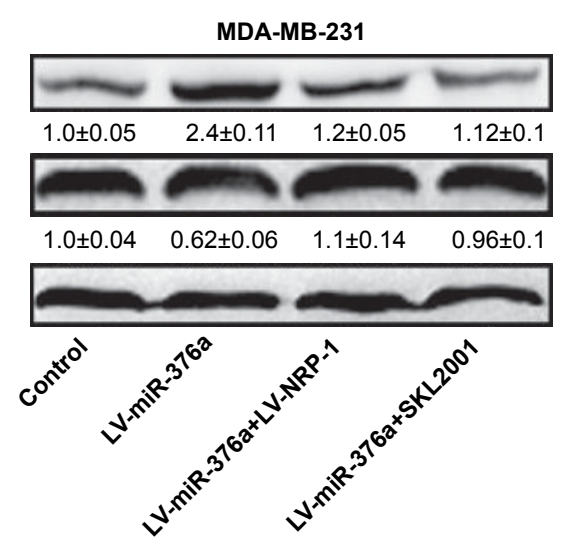

Figure 5 (Continued) 

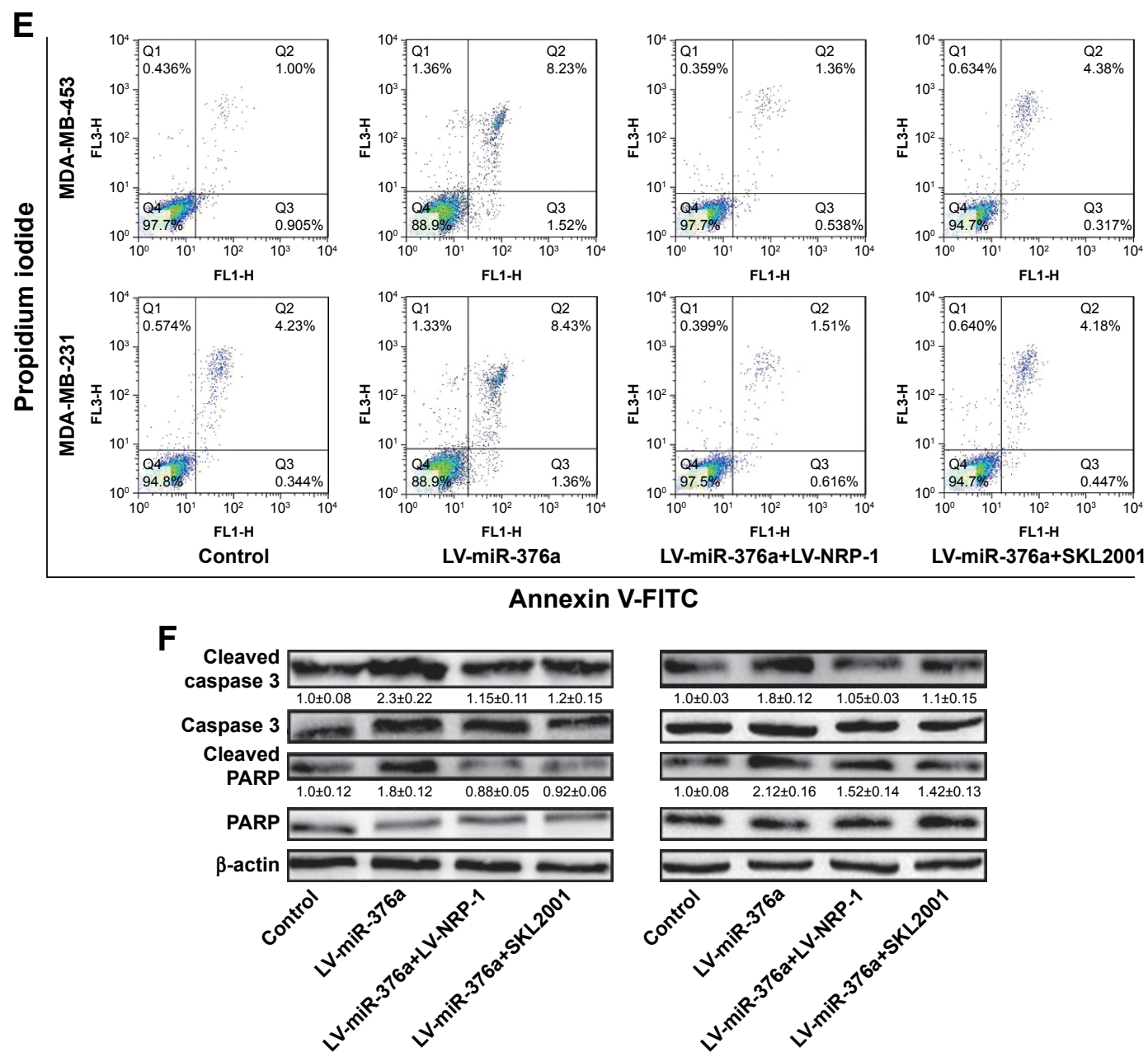

LV-miR-376a+LV-NRP-1 LV-miR-376a+SKL2001

Annexin V-FITC

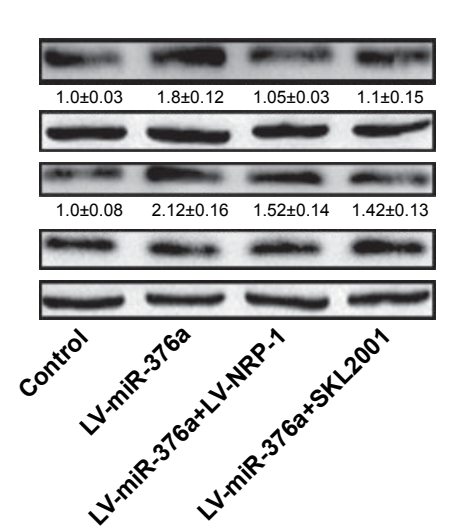

Figure 5 Overexpression of NRP-I or inhibition of Wnt/ $\beta$-catenin signaling rescues the inhibitory effects of miR-376a on breast cancer cell progression.

Notes: (A and B) The cell viability was examined in MDA-MB-453 and MDA-MB-23I cells with miR-376a overexpression as well as NRP-I knockdown or SKL200I treatment. (C) Cell migration ability was detected in cells depicted in (A). (D) The expression of epithelial (E-cadherin) and mesenchymal markers (Vimentin) was measured in cells depicted in (A). (E) Cell apoptosis was evaluated in cells depicted in (A). (F) The expression of apoptosis executors (cleaved caspase 3 and cleaved PARP) was determined in cells depicted in (A). Data are presented as mean $\pm \mathrm{SD} ; * \mathrm{P}<0.05, * * \mathrm{P}<0.0 \mathrm{I}$ vs $\mathrm{NC}$ or control.

Abbreviations: FITC, fluorescein isothiocyanate; h, hours; NRP-I, neuropilin-I; NC, negative control.

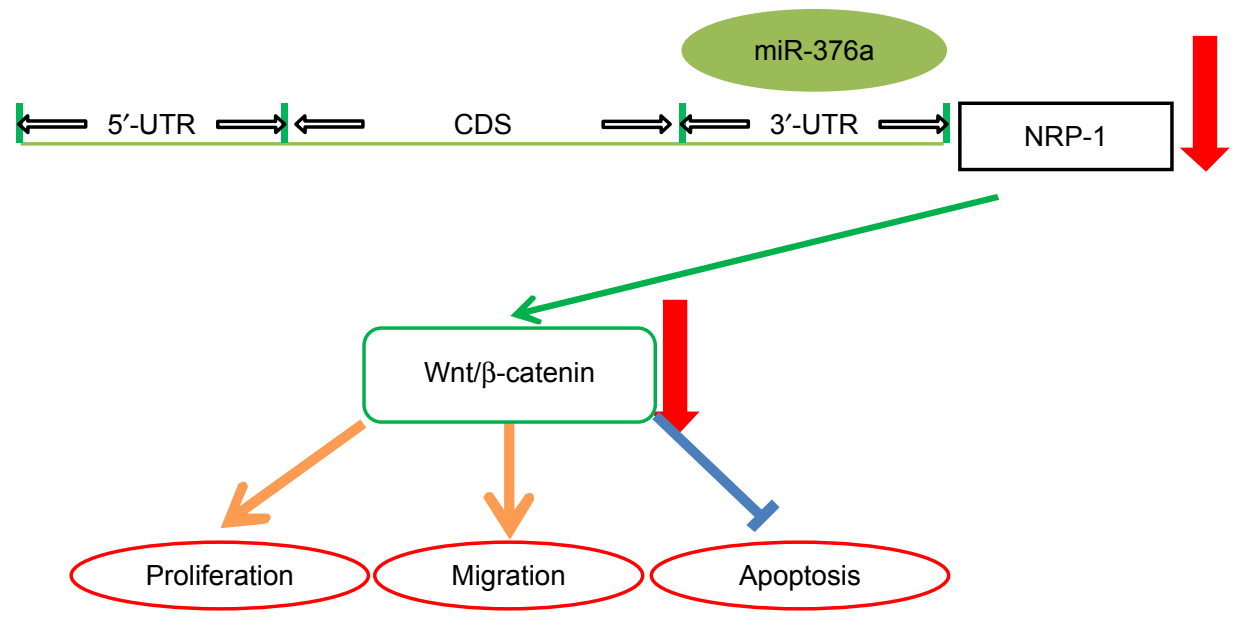

Figure 6 Proposed model that miR-376a inhibits breast cancer cell progression.

Note: miR-376a suppressed breast cancer cell proliferation and migration and promotes cell apoptosis via targeting NRP-I and thus inhibiting Wnt/ 3 -catenin pathway. Abbreviation: NRP-I, neuropilin-I. 


\section{Acknowledgment}

This work was supported by Six Talent Peaks Project in Jiangsu Province (2014-wsw-040).

\section{Disclosure}

The authors report no conflicts of interest in this work.

\section{References}

1. Harbeck N, Gnant M. Breast cancer. Lancet. 2017;389(10074): 1134-1150.

2. Cancer Genome Atlas Network. Comprehensive molecular portraits of human breast tumours. Nature. 2012;490(7418):61-70.

3. Burki TK. Improved progression-free survival in metastatic breast cancer. Lancet Oncol. 2018;19(2):e79.

4. Ni Q, Sun J, Ma C, Li Y, Ju J, Sun M. The neuropilins and their ligands in hematogenous metastasis of salivary adenoid cystic carcinoma-an immunohistochemical study. J Oral Maxillofac Surg. 2018;76(3):569-579.

5. Matkar PN, Rudenko D, Cao WJ, Kuliszewski MA, Prud'homme GJ, Leong-Poi $\mathrm{H}$. Evaluating the therapeutic potential of targeting neuropilins in human pancreatic adenocarcinoma. Mol Ther. 2014;22: S256-S257.

6. O’Byrne K, Gray S, Gately K, Pidgeon G, Barr M. 1061 The neuropilins: critical survival factors for non-small cell lung cancer cells. Eur J Cancer Suppl. 2009;7(2):104.

7. Naik A, Al-Zeheimi N, Bakheit CS, et al. Neuropilin-1 associated molecules in the blood distinguish poor prognosis breast cancer: a cross-sectional study. Sci Rep. 2017;7(1):3301.

8. Luo M, Hou L, Li J, et al. VEGF/NRP-1axis promotes progression of breast cancer via enhancement of epithelial-mesenchymal transition and activation of NF-אB and $\beta$-catenin. Cancer Lett. 2016;373(1):1-11.

9. Starzec A, Ladam P, Vassy R, et al. Structure-function analysis of the antiangiogenic ATWLPPR peptide inhibiting $\operatorname{VEGF(165)~binding~to~}$ neuropilin-1 and molecular dynamics simulations of the ATWLPPR/ neuropilin-1 complex. Peptides. 2007;28(12):2397-2402.

10. Liu WQ, Lepelletier Y, Montès M, et al. NRPa-308, a new neuropilin-1 antagonist, exerts in vitro anti-angiogenic and anti-proliferative effects and in vivo anti-cancer effects in a mouse xenograft model. Cancer Lett. 2018;414:88-98.

11. Zeng F, Luo F, Lv S, et al. A monoclonal antibody targeting neuropilin-1 inhibits adhesion of MCF7 breast cancer cells to fibronectin by suppressing the FAK/p130cas signaling pathway. Anticancer Drugs. 2014;25(6):663-672.

12. Zhang L, Wang H, Li C, et al. VEGF-A/Neuropilin 1 pathway confers cancer stemness via activating Wnt/ $\beta$-Catenin axis in breast cancer cells. Cell Physiol Biochem. 2017;44(3):1251-1262.
13. Han Z, Jiang G, Zhang Y, et al. Effects of RNA interference-mediated NRP-1 silencing on the proliferation and apoptosis of breast cancer cells. Mol Med Rep. 2015;12(1):513-519.

14. Zheng L, Meng X, Li X, et al. miR-125a-3p inhibits ER $\alpha$ transactivation and overrides tamoxifen resistance by targeting CDK3 in estrogen receptor-positive breast cancer. FASEB J. 2018;32(2):588-600.

15. Muhammad N, Bhattacharya S, Steele R, Ray RB. Anti-miR-203 suppresses ER-positive breast cancer growth and stemness by targeting SOCS3. Oncotarget. 2016;7(36):58595-58605.

16. Hu J, Zheng L, Shen X, Zhang Y, Li C, Xi T. MicroRNA-125b inhibits AML cells differentiation by directly targeting Fes. Gene. 2017;620:1-9.

17. Formosa A, Markert EK, Lena AM, et al. MicroRNAs, miR-154, miR299-5p, miR-376a, miR-376c, miR-377, miR-381, miR-487b, miR485-3p, miR-495 and miR-654-3p, mapped to the 14q32.31 locus, regulate proliferation, apoptosis, migration and invasion in metastatic prostate cancer cells. Oncogene. 2014;33(44):5173-5182.

18. Meng X, Joosse SA, Müller V, et al. Diagnostic and prognostic potential of serum miR-7, miR-16, miR-25, miR-93, miR-182, miR-376a and miR-429 in ovarian cancer patients. Br J Cancer. 2015;113(9):1358-1366.

19. Huang Q, Wang C, Hou Z, et al. Serum microRNA-376 family as diagnostic and prognostic markers in human gliomas. Cancer Biomark. 2017;19(2):137-144.

20. Wang Y, Cong W, Wu G, et al. MiR-376a suppresses the proliferation and invasion of non-small-cell lung cancer by targeting c-Myc. Cell Biol Int. 2018;42(1):25-33.

21. Herr I, Sähr H, Zhao Z, et al. MiR-127 and miR-376a act as tumor suppressors by in vivo targeting of COA1 and PDIA6 in giant cell tumor of bone. Cancer Lett. 2017;409:49-55.

22. Györffy B, Lanczky A, Eklund AC, et al. An online survival analysis tool to rapidly assess the effect of 22,277 genes on breast cancer prognosis using microarray data of 1,809 patients. Breast Cancer Res Treat. 2010;123(3):725-731.

23. Yang J, Li T, Gao C, et al. FOXO1 3'UTR functions as a ceRNA in repressing the metastases of breast cancer cells via regulating miRNA activity. FEBS Lett. 2014;588(17):3218-3224.

24. Sheng B, Zhao L, Zang X, Zhen J, Chen W. miR-375 ameliorates sepsis by downregulating miR-2 1 level via inhibiting JAK2-STAT3 signaling. Biomed Pharmacother. 2017;86:254-261.

25. Fellenberg J, Sähr H, Kunz P, et al. Restoration of miR-127-3p and miR-376a-3p counteracts the neoplastic phenotype of giant cell tumor of bone derived stromal cells by targeting COA1, GLE1 and PDIA6. Cancer Lett. 2016;371(1):134-141.

26. Zhang G, Chen L, Khan AA, et al. miRNA-124-3p/neuropilin-1(NRP-1) axis plays an important role in mediating glioblastoma growth and angiogenesis. Int J Cancer. 2018;143(3):635-644.
OncoTargets and Therapy

\section{Publish your work in this journal}

OncoTargets and Therapy is an international, peer-reviewed, open access journal focusing on the pathological basis of all cancers, potential targets for therapy and treatment protocols employed to improve the management of cancer patients. The journal also focuses on the impact of management programs and new therapeutic agents and protocols on

\section{Dovepress}

patient perspectives such as quality of life, adherence and satisfaction. The manuscript management system is completely online and includes a very quick and fair peer-review system, which is all easy to use. Visit http://www.dovepress.com/testimonials.php to read real quotes from published authors. 